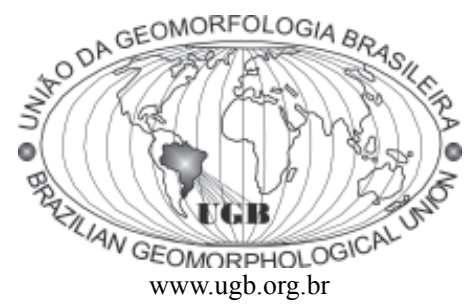

\title{
MODELAGEM DA PRODUÇÃO DE SEDIMENTOS NO NOROESTE FLUMINENSE COMO SUBSÍDIO AO PLANEJAMENTO AMBIENTAL: UM ESTUDO DE CASO DA BACIA DO CÓRREGO SANTA MARIA
}

\author{
Eberval Marchioro \\ Prof. Adj. do Departamento de Geografia - Universidade Federal do Espírito Santo (UFES), Av. Fernando Ferrari, 514, \\ Goiabeiras, Vitória, ES, Brasil - CEP 29075-910 - e-mail: ebervalm@gmail.com \\ Nelson Ferreira Fernandes \\ Prof. Associado do Depto. de Geografia - Universidade Federal do Rio de Janeiro (UFRJ) - Cidade Universitária, \\ Caixa postal 68537, Ilha do Fundão, RJ, Brasil - CEP 21945-970 - e-mail: nelson@pq.cnpq.br \\ José Ronaldo de Macedo \\ Eng. Agrônomo - Pesquisador A - Empresa Brasileira de Pesquisa Agropecuária (Embrapa Solos/RJ), Rua Jardim \\ Botânico, 1.024, Jardim Botânico, Rio de Janeiro, RJ, Brasil - CEP 22460-000 - e-mail: jrmacedo@cnps.embrapa.br \\ Sílvio Barge Bhering \\ Eng. Agrônomo - Pesquisador A - Empresa Brasileira de Pesquisa Agropecuária (Embrapa Solos/RJ), Rua Jardim \\ Botânico, 1.024, Jardim Botânico, Rio de Janeiro, RJ, Brasil - CEP 22460-000 - e-mail: silvio@cnps.embrapa.br
}

Alexandre Ortega Gonçalves

Eng. Agrônomo - Pesquisador B - Empresa Brasileira de Pesquisa Agropecuária (Embrapa Solos/RJ), Rua Jardim Botânico, 1.024, Jardim Botânico, Rio de Janeiro, RJ, Brasil - CEP 22460-000 - e-mail: aortega@cnps.embrapa.br

Marco Aurélio Costa Caiado

Eng. Agrônomo - Prof. Adj. do Inst. Federal do Espírito Santo (IFES-Vitória/ES), Avenida Vitória, 1729, Bairro Jucutuquara, Vitória, ES, Brasil - CEP 29040-780 - e-mail: maccaiado@gmail.com

\begin{abstract}
Resumo
A região noroeste fluminense tem passado por profundas transformações em seu uso e ocupação do solo, aumentando a produção de sedimentos e mudanças da relação solo-água-planta. Em função desse quadro, este estudo visou a analisar a distribuição espacial média da produção de sedimentos na bacia experimental do córrego Santa Maria, no município de São José de Ubá, no noroeste fluminense (RJ), para o período entre 2005 e 2007, com o intuito de subsidiar o planejamento ambiental. Para modelagem da produção de sedimentos foi utilizado o modelo matemático Soil and Water Assessment Tool (SWAT), devido à sua base física e à capacidade de simular cenários ambientais. Para a modelagem foi necessário o Modelo Digital de Elevação (MDE), dados cartográficos de uso e ocupação de solo atual, da espacialização dos solos e suas características físico-hídricas, bem como dados climáticos e hidrossedimentológicos diários e mensais. Os resultados evidenciaram que as áreas com maior produção de sedimentos ocorrem nas Unidades de Respostas Hidrológicas (URHs) com domínio das pastagens associadas às declividades, entre $25 \%$ e $57 \%$, comprimentos de encostas entre 15 e $28 \mathrm{~m}$, e às características do ARGISSOLO VERMELHOAMARELO, NEOSSOLO LITÓLICO (RLve) e CAMBISSOLO HÁPLICO, evidenciando a necessidade de reflorestamento com espécies nativas nas URHs com estas classes de solos e, nas demais porções da bacia do córrego Santa Maria, a difusão de práticas de manejo e conservação do solo.
\end{abstract}


Palavras-chave: Modelagem. Bacia hidrográfica. Produção de sedimentos. Soil and Water Assessment Tool.

\begin{abstract}
The northwestern Rio de Janeiro area has undergone extreme transformations in its soil use and occupation, which has resulted in increased sediment yield and changes in the soil-water-plant relations. Due to this fact, this study aimed at analyzing the average spatial distribution of sediment yield in Santa Maria creek experimental basin, in the county of São José de Ubá, northwestern Rio de Janeiro, between 2005 and 2007, in order to assist in environmental planning. The mathematical model Soil and Water Assessment Tool (SWAT) was used for sediment yield modeling because of its physical basis and capacity to simulate environmental scenarios. The Digital Elevation Model (DEM), cartographic data on the current soil use and occupation, soil distribution and physical and hydrological characteristics, as well as daily and monthly climate and hydrosedimentologic data were used in the modeling. The results showed that the areas with higher sediment yield are located in the Hydrological Response Units (HRUs) predominantly made up of pastures associated with slopes of 25 to $57 \%$; slope lengths between 15 and $28 \mathrm{~m}$; and RED-YELLOW LOAMY SOIL, LITHOLIC NEOSOL and HAPLIC CAMBISOL. This shows that the HRUs that have these classes of soil need to be reforested with native species, and in other parts of Santa Maria creek basin, management and soil conservation practices should be promoted.
\end{abstract}

Keywords: Modeling. Hydrographic basin. Sediment yield. Soil and Water Assessment Tool.

\section{Introdução}

Compreender os fatores ambientais que influenciam a produção de sedimentos em uma bacia hidrográfica é importante para o planejamento ambiental, pois possibilita mitigar as áreas fonte e os seus fatores controladores. Isso contribui, por exemplo, para minimizar as taxas de produção de sedimentos, aumentar a vida útil de reservatórios e minimizar o assoreamento dos cursos fluviais (FOX et al., 1997; LANE et al., 1997).

A produção de sedimentos é um processo natural decorrente da interação entre as características dos solos, da cobertura vegetal, do uso e ocupação do solo, da topografia e da distribuição e intensidade das chuvas, entre outros elementos que variam no tempo e no espaço (MORGAN, 1980; HADLEY et al.,1985). Apesar de ocorrer naturalmente, esse processo tem sido afetado pela interferência antrópica, acelerando em até uma escala de magnitude a produção de sedimentos nas regiões tropicais montanhosas (HEWAWASAMET et al., 2003; VANACKER et al., 2007).

Tradicionalmente, os estudos da produção de sedimentos foram realizados por meio de parcelas experimentais instaladas nas encostas, com o intuito de verificar o impacto da ação antrópica, das práticas de manejo e conservação do solo e desenvolver e/ou avançar tecnologias no controle de erosão dos solos, entre outros (De PLOEY; GABRIELS, 1980; CERDAN et al., 2004; BOIX-FAYOS et al., 2006). Dentro desse contexto, Stroosnijder (2005) destaca que existem escalas relevantes para análise da erosão dos solos: a) escala de $1 \mathrm{~m}^{2}$, para medir o efeito do respingo; b) escala $\leq 100 \mathrm{~m}^{2}$, utilizada para monitorar erosão de solos nas encostas; c) escala de $\leq 500 \mathrm{~m}^{2}$ para verificar a erosão e deposição na encosta; d) escala $<1$ ha para canais; e e) escala $<50$ ha para verificar o efeito das interações espaciais em pequenas bacias hidrográficas. Apesar das escalas sugeridas, no Brasil, trabalhos conduzidos por Araújo-Neto (1993), Guerra (1995), Baccaro (1999), Marchioro e Augustin (2007), entre outros, valeram-se de parcelas experimentais de $10 \mathrm{~m}^{2}$ a $100 \mathrm{~m}^{2}$ para monitorar a erosão dos solos.

Apesar das informações relevantes obtidas por meio das parcelas experimentais, De Vente e Poesen (2005) salientam que esses experimentos não são representativos de todos os processos envolvidos para a produção de sedimentos na escala de bacia hidrográfica. Em função desse quadro, na atualidade tem sido desenvolvido estudo de produção de sedimentos em bacias hidrográficas, utilizando-se de modelos matemáticos de bases físicas, que incorporaram o Sistema de Informação Geográfica (SIG), permitindo capturar, manipular, processar dados e efetuar a transposição de escala espacial e temporal (BEVEN, 2001; MERRIT et al., 2003; AKSOY; KAVVAS, 2005).

Dentre os inúmeros modelos existentes, pode-se destacar a Equação Universal de Perda de Solo (USLE), o modelo Chemicals, Runoff and Erosion from Agricultural Management Systems Models (CREAMS) (KNISEL, 1980), o Areal Nonpoint Source Watershed Environmental Response Simulation (ANSWERS) (BEASLEY et al., 1980), Erosion Productivity Management Systems (EPIC) (WILLIAMS et al., 1984), Water Erosion Prediction Project (WEPP) (FLANAGAN et al., 2007) e Simulator for Water Resources in Rural Basins (SWRRB) (WILLIAMS et al.,1985; ARNOLD et al.,1990).

A continuidade dos esforços para aprimorar e/ou desenvolver novos modelos capazes de analisar a influência das formas de uso e ocupação do solo, das práticas de manejo e conservação em bacias hidrográficas, levou à elaboração do Soil and Water Assessment Tool (SWAT) (ARNOLD; SRINIVASAN, 1995). Esse modelo possui: a) capacidade 
de simular produção de sedimentos de modo contínuo para bacia, permitindo, assim, a simulação para longos períodos (>100 anos) ou para um dia; b) poder de simular transporte e deposição de sedimentos no canal; e c) capacidade de elaborar cenários ambientais.

Em função das possibilidades oferecidas pelo SWAT, esse modelo foi utilizado neste trabalho para: a) analisar a distribuição espacial da produção de sedimentos na bacia do córrego Santa Maria no município de São José de Ubá, no noroeste fluminense, para o período entre 2005 e 2007, com o intuito de subsidiar o planejamento ambiental; e b) confrontar os resultados de Concentração de Sólidos em Suspensão (CSS) - gerados pelo modelo SWAT com os mensurados na bacia de drenagem durante o período entre dezembro de 2006 e dezembro de 2007.

\section{Área de Estudo}

A bacia do córrego Santa Maria localiza-se no município de São José de Ubá, na região noroeste do Estado do Rio de Janeiro, que é denominada de Noroeste Fluminense, apresentando uma área de 1.356ha, como pode ser visto na Figura 1. É integrante de um conjunto de sub-bacias que drenam suas águas para a bacia do rio Paraíba do Sul, um dos mais importantes sistemas hidrográficos do Sudeste do Brasil.

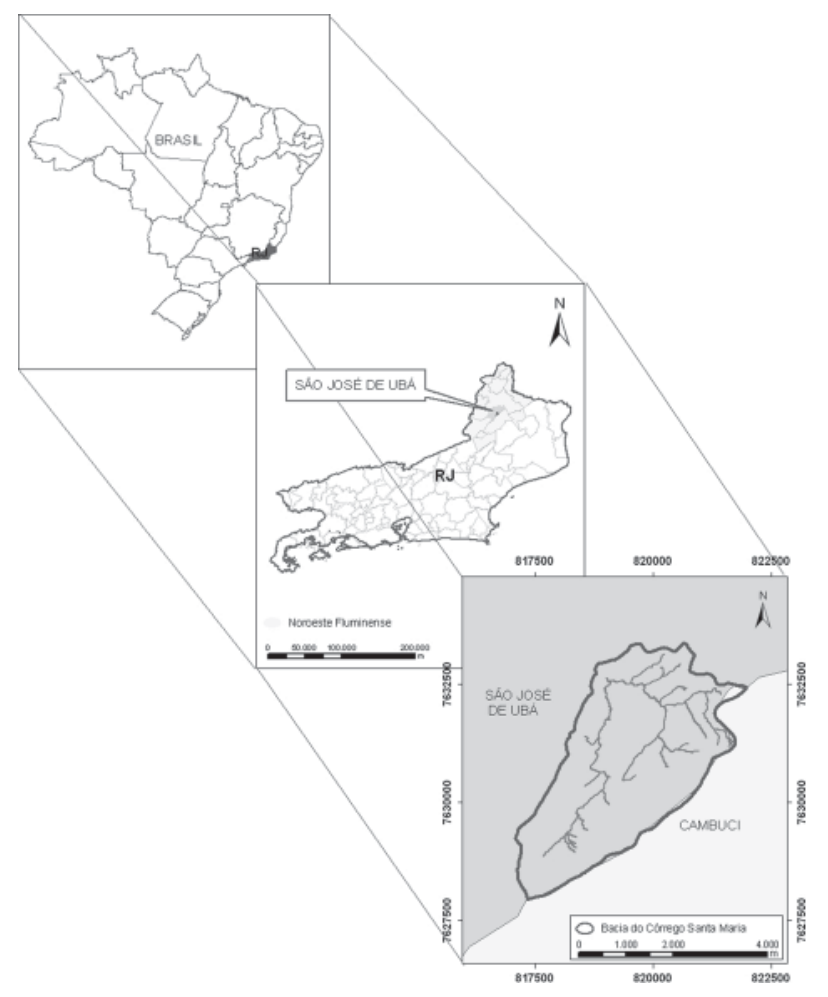

Figura 1 - Localização geográfica do município de São José de Ubá no noroeste do Rio de Janeiro e da bacia experimental do córrego Santa Maria.
O clima de São José de Ubá foi caracterizado por Gonçalves et al. (2006) como do tipo Aw, ou seja, tropical quente e úmido com estações climáticas bem definidas. Baseado em dados de 2005 a 2007 coletados na bacia, verifica-se que a precipitação média anual é de $1.283,72 \mathrm{~mm}$. O trimestre consecutivo mais chuvoso é de novembro a janeiro, totalizando $663,29 \mathrm{~mm}$, o equivalente a $51,66 \%$ do total pluviométrico anual. O mês mais chuvoso é janeiro, com $314,38 \mathrm{~mm}$, representando $24,48 \%$ do total pluviométrico anual. $\mathrm{O}$ trimestre consecutivo seco ocorre entre o período de junho, julho e agosto, com índices pluviométricos de $28,17 \mathrm{~mm}, 28,34 \mathrm{~mm}$ e $7,28 \mathrm{~mm}$ respectivamente, representando, em termos percentuais, o equivalente a $4,97 \%$ do índice pluviométrico médio do período citado (MARCHIORO, 2008). A temperatura média para o período de 1960-1990 foi de $23,1^{\circ} \mathrm{C}$, sendo o mês de fevereiro, $\operatorname{com} 26,4^{\circ} \mathrm{C}$, o mais quente, e o de junho, o mais frio, com $19,9^{\circ} \mathrm{C}$. O trimestre mais quente é o compreendido entre janeiro e março, e o mais frio entre junho e agosto (GONÇALVES et al., 2006).

O substrato da bacia do córrego Santa Maria é composto pelas unidades geológicas São José de Ubá e Vista Alegre (Complexo Juiz de Fora), Catalunha (Complexo Paraíba do Sul) e os Sedimentos Quaternários (REIS; MANSUR, 1995). A primeira é formada por hiperstênio, possuindo características metamórficas, com granulação fina, coloração cinza-esverdeada, maciça e foliada. A segunda é composta por uma complexa associação entre migmatitos e rochas metamórficas extremamente heterogêneas. A unidade Catalunha é constituída por gnaisses, às vezes com grafita e cordierita. Os Sedimentos Quaternários são de origem fluvial, situados nas planícies de inundação (REIS; MANSUR, 1995).

A bacia possui importante descontinuidade geológica longitudinal, com rumo NE-SW, e as rochas encontram-se cortadas por um conjunto de fraturas, predominando os trends NNW-SSE e NE-SW (concordante com a estruturação geológica da bacia) concentradas na parte norte da bacia (Domínio Juiz de Fora), devido à heterogeneidade das rochas e uma estruturação milonítica mais pronunciada (HEILBRON et al., 2005).

A bacia está inserida na unidade geomorfológica Depressão Interplanáltica com Alinhamentos Serranos do Norte-Noroeste Fluminense (DANTAS, 2000). Essa unidade caracteriza-se por apresentar contraste brusco entre feições homogêneas de colinas, morros e morrotes associados aos alinhamentos serranos elevados.

As classes de solos predominantes na bacia são os CAMBISSOLOS HÁPLICOS lépticos que abrangem 44\% da área, os ARGISSOLOS VERMELHO-AMARELO abrangendo uma área de 18,5\% e os NEOSSOLOS LITÓLICOS com uma área 16,7\%. Em conjunto, totalizam 79,2 da área da bacia. Os restantes $20,8 \%$ são compostos, em ordem decrescente de área, pelos CAMBISSOLOS HÁPLICOS gleicos, GLEISSOLOS HÁPLICOS e Afloramento de rocha (BHERING et al., 2005; ZARONI, 2006). 


\section{Materiais e Métodos}

Para a execução da modelagem da produção de sedimentos, foi escolhido a bacia hidrográfica experimental do córrego Santa Maria, já que apresenta, em termos geológicos, geomorfológicos e de uso e ocupação do solo, características representativas do noroeste fluminense.

Outro fato que contribuiu para a modelagem da produção de sedimentos e da concentração de sólidos em suspensão na água (CSS), no exutório da bacia hidrográfica do córrego Santa Maria, foi a disponibilização do banco de dados pelo projeto "Planejamento conservacionista das terras e modelagem preditiva de sistemas aquíferos do cristalino, para recarga hídrica em bacias hidrográficas de relevo acidentado", liderado pela Empresa Brasileira de Pesquisa Agropecuária (Embrapa - RJ).

\section{Estratégias da modelagem da produção de sedimentos e do monitoramento de concentração de sólidos em suspensão (CSS) na bacia}

O modelo utilizado para a simulação foi o Soil and Water Assessment Tool (SWAT), versão 2005, denominada AVSWAT-X que, originalmente, foi proposto no início da década de 1990 por Jeff Arnold e tem sido mantido e aperfeiçoado pelo United States Department - Agricultural Research Service (USDA - ARS) e pelo Soil and Water Research Laboratory -Temple-Texas, nos Estados Unidos da América (EUA).

Os componentes do modelo incluem aspectos hidrológicos, climáticos, sedimentológicos, de crescimento das plantas, nutrientes, pesticidas e de manejo agrícola. $\mathrm{O}$ componente hidrológico do modelo inclui sub-rotinas do escoamento superficial, percolação, fluxo lateral subsuperficial, fluxo de retorno do aquífero raso e evapotranspiração (NEITSCH et al., 2005).

A produção do escoamento superficial é estimada pelo modelo SWAT por meio da informação da curva número de cada tipo de uso e ocupação do solo e sua prática de manejo e conservação (NEITSCH et al., 2005). O SWAT utiliza a Equação Universal de Perda de Solo Modificada - MUSLE (WILLIAMS, 1995) para estimar a produção de sedimentos, levando em consideração a força do escoamento nas vertentes e no canal fluvial. Para a simulação do volume e da velocidade da água no canal, o modelo SWAT utiliza a equação de Manning's e o tempo de propagação é computado pela divisão do comprimento do canal pela velocidade (NEITSCH et al., 2005). A interface do modelo com o Sistema de Informação Geográfica (SIG) (ArcView 3.2) permite que as saídas do modelo sejam exibidas em mapas e gráficos (NEITSCH et al., 2005).
Os dados necessários para modelagem da produção de sedimentos na bacia hidrográfica do córrego Santa Maria são o Modelo Digital de Elevação (MDE), o mapa de uso e ocupação do solo, o mapa pedológico com suas características físico-hídricas e os dados climáticos diários e mensais que foram inseridos ao modelo SWAT por meio da sua interface com o ArcView 3.2 desenvolvido por Di Luzio et al. (2002), como pode ser visto na Figura 2.

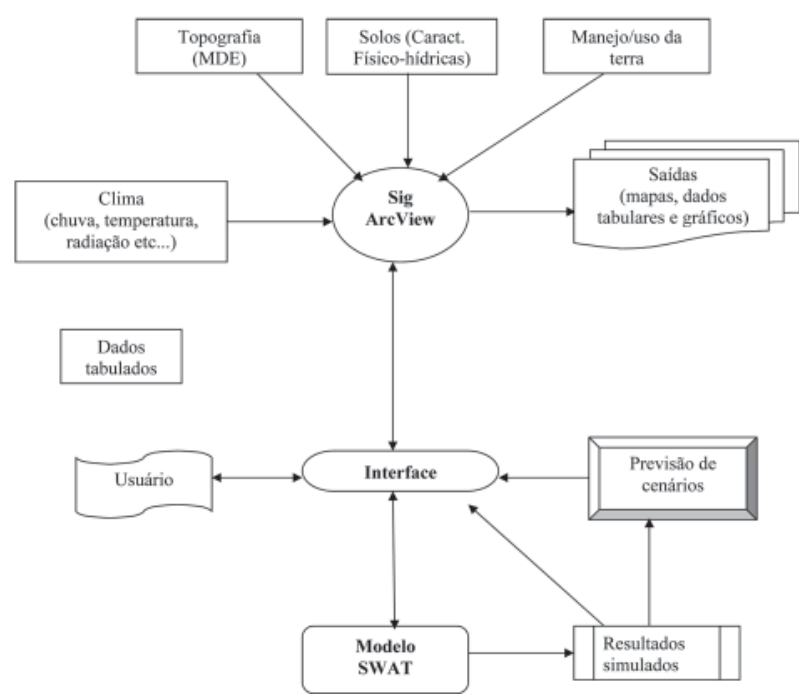

Figura 2 - Diagrama da metodologia utilizada para geração do banco de dados e simulações do modelo SWAT na interface SIG. Fonte: NEITSCH et al. (2005).

Para a elaboração do Modelo Digital de Elevação (MDE), foi realizada a restituição planialtimétrica, na escala de 1:10.000, de fotografias aéreas pancromáticas na escala de 1:20.000 e 1:30.000 da Companhia de Eletricidade do Rio de Janeiro (CERJ) por Fidalgo e Abreu (2005). A equidistância das curvas utilizadas no mapa de entrada na interface do SWAT foi de $30 \mathrm{~m}$.

Uma vez inserido o MDE, o modelo SWAT subdivide automática ou manualmente as sub-bacias ou Unidades de Respostas Hidrológicas (URHs), exportando relatório para cada uma delas. A definição da área mínima de cada sub-bacia para o desenvolvimento deste trabalho foi realizada por meio manual, considerando a comparação entre a drenagem obtida no ArcView 9.1 por Zaroni (2006) e a drenagem previamente definida pela interface do SWAT.

Finalizado tal processo, foram introduzidos o mapa de uso e ocupação do solo e o mapa pedológico. O mapa de uso e ocupação do solo atual da bacia do córrego Santa Maria foi elaborado preliminarmente por Zaroni (2006), utilizando fotografias aéreas do tipo pancromáticas, na escala de 1:20.000 e 1:30.000 de 2000, fornecidas pela CERJ. Para esse mapeamento, usou-se a classificação automática 
utilizando-se o SIG e o programa computacional SPRING 4.1, com posterior validação em campo. Contudo, foi verificada no mapeamento uma faixa da bacia do córrego Santa Maria que não havia sido recoberta pelo sobrevoo, culminando na ausência de dados nessa área do mapa.

Para complementar o mapeamento supracitado, realizou-se fotointerpretação visual dessa área não recoberta pelo sobrevoo da CERJ, utilizando-se o ArcGis 9.1. Para tal, foram usadas fotografias aéreas disponibilizadas pelo Departamento de Recursos Minerais (DRM/RJ), do voo da Força Área Brasileira (FAB) de 1976, na escala de 1:10.000, e posterior validação do mapeamento em campo da área preenchida manualmente. É importante salientar que, na faixa preenchida manualmente, não foi possível manter a escala obtida na classificação automática e a riqueza de detalhes, uma vez que se buscou contornar polígonos com maior representatividade espacial (MARCHIORO, 2008).

O mapa de solos da bacia do córrego Santa Maria e os respectivos parâmetros físico-hídricos de cada classe de solo dominante nas unidades de mapeamento foram fornecidos por Bhering et al. (2005) e introduzidos ao modelo SWAT por meio da interface ArcView 3.2.

Uma vez introduzidos os dados cartográficos, MDE, de uso e ocupação do solo e o mapa pedológico, as Unidades de Respostas Hidrológicas (URHs) foram definidas, levando-se em consideração o seu uso e ocupação do solo dominante.

Para a elaboração das simulações, o modelo requer um banco de dados climáticos composto por dados diários de chuva (obrigatório), temperatura (obrigatório), radiação solar (modelável), umidade relativa do ar (modelável) e velocidade do vento (modelável) e a criação de uma estação meteorológica com dados médios mensais. A criação dessa estação foi feita na interface do SWAT com o ArcView 3.2.

Para suprir as necessidades das informações climáticas, foram utilizados dados diários de precipitação para o período de $1^{\circ}$ de janeiro de 2005 a 31 de dezembro de 2007, obtidos por uma estação termopluviométrica localizada nas coordenadas UTM de 0196838 e 7632887, Datum WGS 1984, Fuso 24S, situada a uma altitude de 149 metros no exutório bacia do córrego Santa Maria. Para a temperatura máxima e mínima, umidade relativa e velocidade do vento, foram utilizados dados diários da estação meteorológica automática de Itaperuna/RJ, localizada entre as latitudes $21^{\circ} 12^{\prime} \mathrm{S}$ e $41^{\circ} 54^{\prime} \mathrm{W}$, situada a uma altitude de 123 metros, que foram disponibilizados pelo Instituto Nacional de Meteorologia (Inmet) de Brasília. A radiação foi modelada pelo SWAT e o método utilizado para o cálculo da evapotranspiração foi o Penman-Montheith.

Também, para a realização da modelagem, criou-se uma estação meteorológica na interface ArcView 3.2 com o SWAT, com os seguintes parâmetros: média mensal da temperatura máxima do ar $\left[{ }^{\circ} \mathrm{C}\right]$; média mensal da temperatura mínima do ar $\left[{ }^{\circ} \mathrm{C}\right]$; desvio padrão para temperatura máxima mensal do ar $\left[{ }^{\circ} \mathrm{C}\right]$; desvio padrão para temperatura mínima mensal do ar $\left[{ }^{\circ} \mathrm{C}\right]$; média da precipitação mensal $[\mathrm{mm}]$; desvio padrão para precipitação diária neste mês $[\mathrm{mm}]$; coeficiente de distorção da chuva diária (Skew coefficient); probabilidade de um dia chuvoso seguido de um dia seco mensal; probabilidade de um dia chuvoso seguido de outro dia chuvoso mensal; média mensal dos dias chuvosos; chuva máxima no período de 30 minutos [mm] e seu período de retorno [anos]; média mensal da radiação solar diária $\left[\mathrm{MJ} / \mathrm{m}^{2} \mathrm{xdia}\right]$; média mensal do ponto de orvalho $\left[{ }^{\circ} \mathrm{C}\right]$; e média mensal da velocidade do vento $[\mathrm{m} / \mathrm{s}]$.

Para a calibração e validação do modelo SWAT, foram utilizados dados de concentração de sólidos em suspensão (CSS) na água do exutório da bacia do córrego Santa Maria. Os dados de CSS foram obtidos pela estação automática Solar 2000MIM que está localizada na mesma posição geográfica da estação termopluviométrica.

A estação Solar 200 MIM é constituída de um datalogger e um sensor de turbidez do tipo SL2000-TS, que estima a CSS diária. O sensor de turbidez mede a frequência da luz que o retorna após a reflexão pelos sedimentos em suspensão na água à sua frente, na faixa de luz infravermelho entre $800 \mathrm{a}$ $860 \mathrm{~nm}$. Essa mudança da frequência da luz é captada e interpretada pelo sensor e transmitida na forma de sinais elétricos ao datalogger, que os converte em dados de CSS.

A calibração do SWAT foi feita de forma automática, utilizando-se dados de concentração de sólidos em suspensão (CSS) diário, para o período de abril a dezembro de 2005, e validação de dezembro de 2006 a setembro de 2007. Para avaliar a eficiência do SWAT em simular a CSS, utilizou-se o Coeficiente de Eficiência de Nash-Sutcliffe (COE), podendo variar desde menos infinito até 1 , sendo 1 o indicativo de simulação perfeita do evento simulado em relação ao medido.

Os resultados das simulações do SWAT são apresentados neste trabalho por meio de mapas, gráficos e tabelas para as Unidades de Respostas Hidrológicas (URHs) ou sub-bacia.

\section{Resultados e Discussão}

\section{Validação da concentração de sólidos em suspensão (CSS) no exutório da bacia}

Em relação à concentração de sólidos em suspensão na água, na Tabela 1 são apresentados os resultados da simulação com e sem calibração, utilizando o uso e ocupação do solo atual, assim como os valores observados no exutório da bacia do córrego Santa Maria para o mesmo período.

Pode-se constatar, na Tabela 1, que os valores simulados com e sem calibração de CSS para os meses secos foram praticamente nulos, devido aos baixos índices pluviométricos 
que, consequentemente, ocasionaram pouco escoamento superficial e baixa concentração de sólidos em suspensão para essa época do ano, em relação aos valores observados. Contudo, também na Tabela 1, é possível verificar que, no mês de janeiro, para a modelagem com e sem calibração, o modelo SWAT estimou uma CSS na água superior ao observado em campo. Para a modelagem com calibração a diferença em relação aos dados monitorados foi de 24,2872mg.L-1 ${ }^{-1}$, mostrando uma alta sensibilidade do SWAT à concentração das chuvas no mês janeiro.

Tabela 1 - Concentração de sólidos em suspensão (mg.L ${ }^{-1}$ ) no exutório da bacia do córrego Santa Maria, no noroeste fluminense (RJ).

\begin{tabular}{c|c|c|c}
\hline Meses & Monitorado & Sem Calibração & Calibrado \\
\hline Dez & 12,3267 & 28,52880 & 6,2765 \\
\hline Jan (2007) & 11,6498 & 51,43182 & 35,9370 \\
\hline Fev & 12,3260 & 1,78500 & 0,0027 \\
\hline Mar & 11,4858 & 21,5300 & 6,6460 \\
\hline Abr & 12,8924 & 10,4440 & 2,0910 \\
\hline Mai & 11,0714 & 0,0493 & 0,0139 \\
\hline Jun & 8,4352 & 0,1840 & 0,0517 \\
\hline Jul & 8,4835 & 0,4384 & 0,1504 \\
\hline Ago & 6,7005 & 0,1291 & 0,3156 \\
\hline Set & 5,1118 & 0,1237 & 0,1270 \\
\hline Out & 2,9560 & 0,24896 & 0,24579 \\
\hline Nov & 3,4766 & 0,1581 & 0,1197 \\
\hline Dez & 4,0216 & 3,9430 & 1,7790 \\
\hline COE & - & $-12,4516$ & $-6,1136$ \\
\hline
\end{tabular}

Os valores de COE obtidos para as simulações sem e com calibração de CSS foram negativos, respectivamente, $-12,4516$ e - 6,1136, evidenciando que a variância interna dos valores simulados pelo modelo SWAT de 184,6885 sem calibração e, 97,6681 calibrado, foi superior aos monitorados.

Considerando os dados de concentração de sólidos em suspensão monitorados e simulados para a bacia do córrego Santa Maria, verificou-se uma discrepância de 57,1813mg.L $\mathrm{L}^{-1}$ entre os valores monitorados em campo e os simulados após a calibração do modelo SWAT.

Esta discrepância foi ocasionada pelo efeito do sistema de monitoramento, associado à forma de obtenção dos dados e às constantes falhas do sensor de CSS. Além disso, o local de instalação do sensor passou a ser utilizado para lazer devido ao represamento da água, promovendo a movimentação dos sedimentos de fundo do canal, contribuindo para superestimar os dados medidos no período seco, como pode ser visto na Figura 3 e na Tabela 1. Em função desses dados de entrada, o SWAT estimou uma incerteza de $97,5 \%$ para o ano 2005 utilizado para calibração, refletindo-se sobre o ajuste do modelo para posterior validação entre os anos de 2006 e 2007.

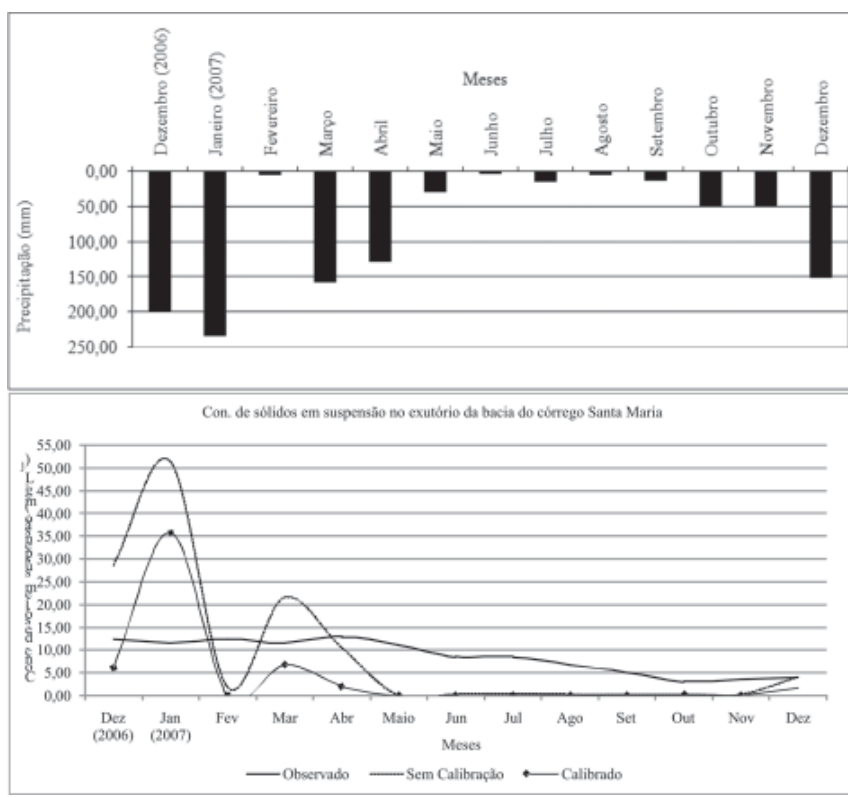

Figura 3 - Comportamento sazonal da concentração de sólidos em suspensão observada e simulada da bacia do córrego Santa Maria para o período entre 2006 a 2007.

Minotti (2006), empregando o SWAT no curso médio e superior da bacia do rio Mogi em São Paulo, obteve também um COE de apenas 0,33, derivado do frágil banco de dados utilizado para calibração e validação.

\section{A produção de sedimentos nas URHs da bacia do córrego Santa Maria prevista pelo SWAT}

Para analisar a distribuição espacial da produção de sedimentos por URHs da bacia do córrego Santa Maria, com o intuito de subsidiar o planejamento ambiental, considerou-se a simulação após a calibração do SWAT, já que apresentou melhor COE com referência a sem calibração.

Na Figura 4, observa-se que a sub-bacia de número 9, apresentou a maior de produção de sedimentos média na bacia do córrego Santa Maria, para o período entre 2005 e 2007, simulada pelo SWAT, que foi de 16,517 t ha-1. Essa bacia apresentou uso e ocupação do solo de pastagem, classe de solo ARGISSOLO VERMELHO-AMARELO, declividade média de $25 \%$ e comprimento de encosta médio de $15,24 \mathrm{~m}$.

Apesar desta sub-bacia ou URHs de número 9, apresentar a maior produção de sedimentos média da bacia do córrego Santa Maria, observa-se na Figura 5, que as principais áreas fonte de sedimentos da bacia estão entre as URHs de número 51 e 57, que apresentam declividade média de $48,77 \%$, associada ao uso e ocupação do solo dominante de pastagem e classes de 
solo RLVE (NEOSSOLO LITÓLICO - Eutrófico típico, A moderado, textura média) e CXve2 (CAMBISSOLO HÁPLICO - Ta, Eutrófico léptico, A moderado, textura média/argilosa).

No que se refere à distribuição espacial da produção de sedimentos na bacia do córrego Santa Maria, pode-se observar, na Figura 6, que, nas URHs de número 34, 44, $48,51,52,54$ e 56 com classe dominante de solos RLVE (NEOSSOLO LITÓLICO - Eutrófico típico, A moderado, textura média) e às URHs de número 38, 39, 45 e 53 com classe de solo CXve2 (CAMBISSOLO HÁPLICO - Ta, Eutrófico léptico, A moderado, textura média/argilosa) e com predomínio de pastagem, a declividade contribuiu para aumentar a produção de sedimentos, de modo que a URHs de número 54 , com $57,40 \%$ de declividade ficasse entre as maiores produtoras de sedimentos dentre as demais na bacia.

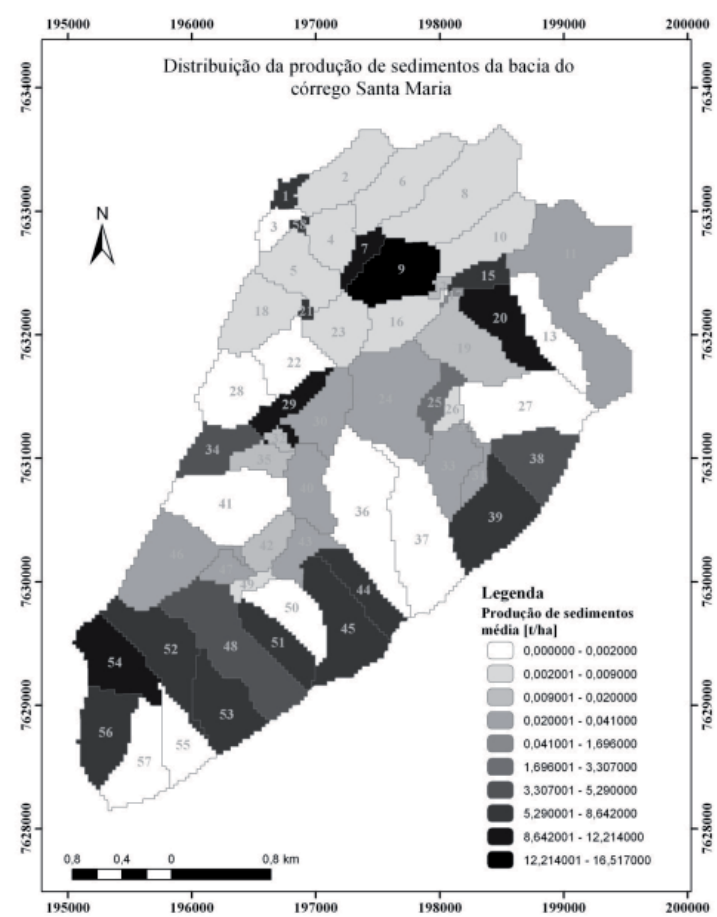

Figura 4 - Mapa da distribuição média da produção de sedimentos média para o período entre 2005 e 2007 na bacia do córrego Santa Maria no Noroeste fluminense.

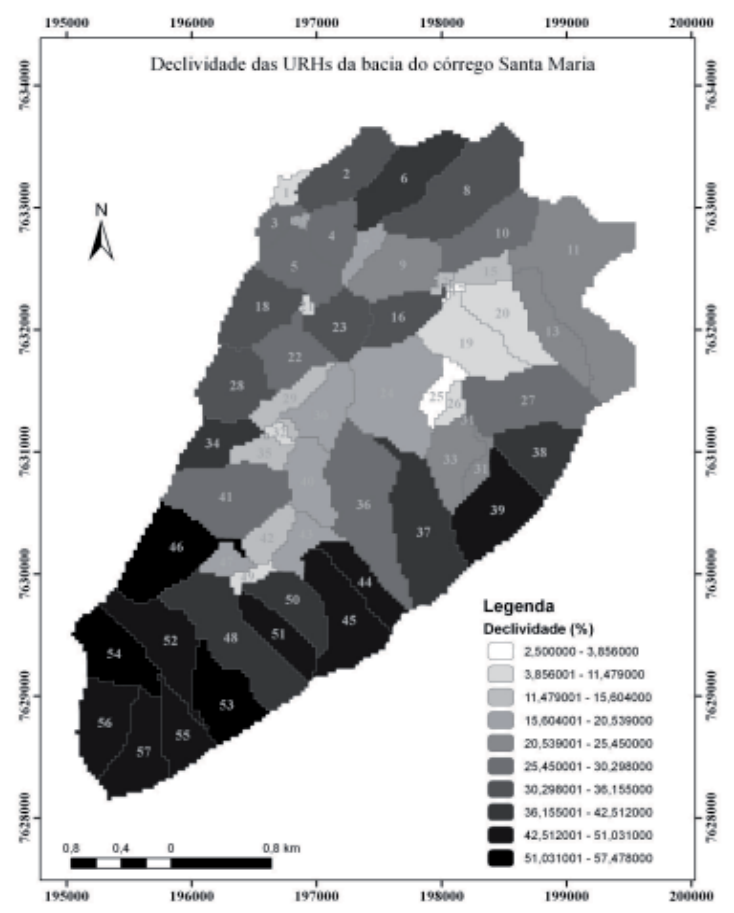

Figura 5-Mapa de declividade das Unidades de Respostas Hidrológicas utilizado pelo modelo SWAT na modelagem hidrossedimentológica.

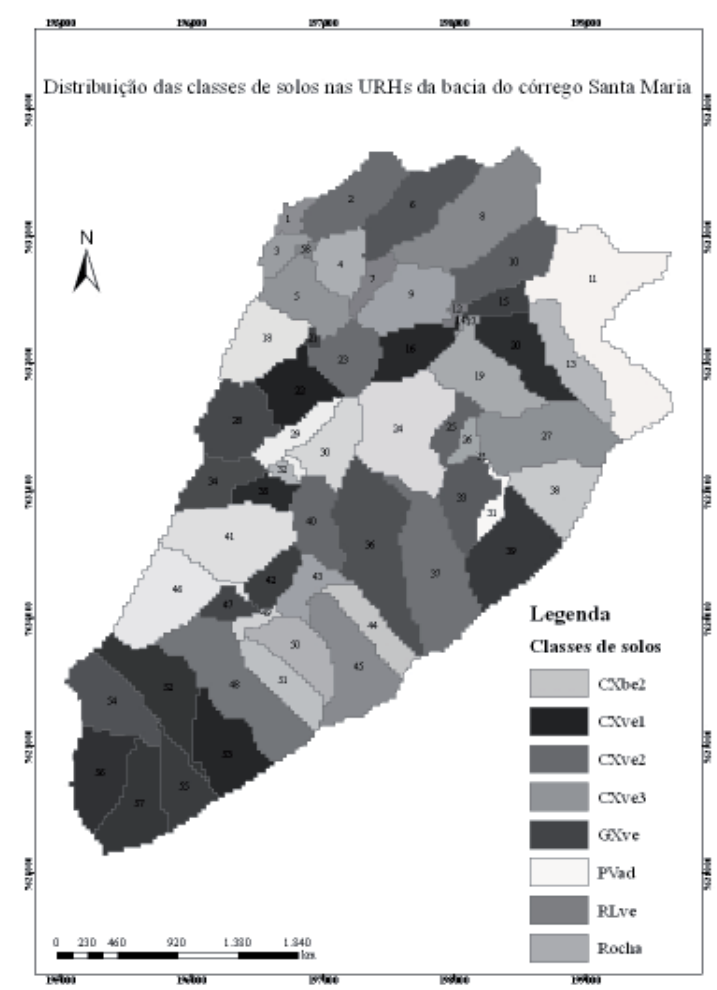

Figura 6 - Mapa das classes de solos das Unidades de Respostas Hidrológicas utilizado pelo modelo SWAT na modelagem hidrossedimentológica. 
Apesar do verificado anteriormente, as URHs de número 55 e 57 com classes de solo RLve e CXve2 e declividade de $49,10 \%$ e $48,20 \%$, respectivamente, não seguiram a mesma tendência, pois sua produção de sedimentos simuladas pelo modelo SWAT foi nula. Isso ocorreu devido ao fato de essas URHs apresentarem cobertura vegetal dominante de Mata Atlântica, o que contribuiu para minimização do efeito do respingo, aumento da infiltração de água no solo e minimização das taxas de escoamento superficial, como é destacado por Carson e Kikby (1972) e Coelho Neto $(1987,1995)$, entre outros.

Nas URHs de número 1, 3, 4, 5, 7, 8, 9, 10, 15, $16,18,23,29,58$ associadas à classe dominante de solo PVad (ARGISSOLO VERMELHO-AMARELO - Distrófico típico, A moderado, textura média/argilosa), às de número $11,13,22,24,27,28,30,31,32,33,35,36$, $37,40,41,43,47$ e 50 , onde a classe dominante do solo é CXve ${ }_{3}$ (CAMBISSOLO HÁPLICO - Tb, Eutrófico típico, A moderado, textura média, fase floresta tropical subcaducifólia relevo ondulado) e às URHs de número12, 14, 19, 26, 42 e 49, com classe de solo dominante CXve1 (CAMBISSOLO HÁPLICO Ta, Eutrófico gleico, A moderado, textura média) e o predomínio de uso e ocupação do solo de pastagem e, comprimento de encostas oscilando entre $15 \mathrm{~m}$ e $25 \mathrm{~m}$ (Figura 7) ocorreram as maiores taxas de produção de sedimentos, sendo que, para comprimentos maiores ou menores nessas URHs citadas, a produção de sedimentos foi minimizada.

Para as URHs de número 17, 20, 21 e 25 onde a classe de solo dominante é o GXve (GLEISSOLO HÁPLICO - Ta Eutrófico típico, A moderado, textura média/argilosa) com predomínio da pastagem, a maior produção de sedimentos esteve associada ao comprimento da encosta de $36,59 \mathrm{~m}$ e para valores maiores, ocorrem também, a sua diminuição.

A tendência entre o aumento da declividade e a produção de sedimentos observada em algumas URHs é compatível com o proposto por Morgan (1980) e por Hadley et al. (1985), que salientam que o acréscimo na declividade contribui para o aumento na velocidade do escoamento superficial e sua capacidade de erosão. Contudo, Evans (1980), Carson e Kirkby (1972), entre outros, salientam que é necessário ter cuidado com essa tendência, visto que, em severas declividades, é possível haver diminuição da erosão em função do decréscimo do material disponível.

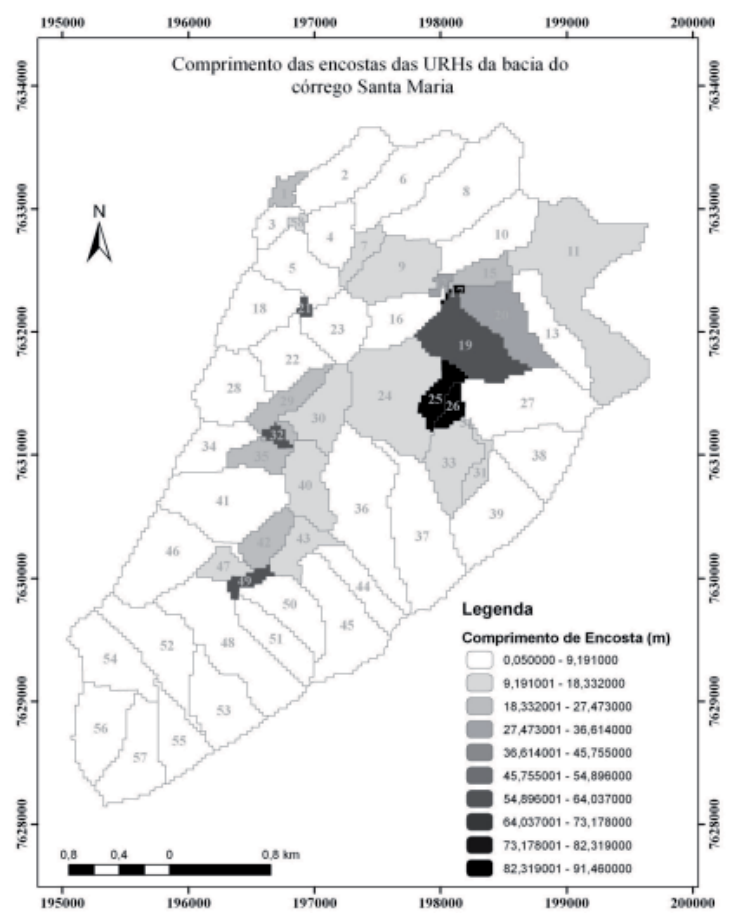

Figura 7 - Mapa do comprimento das encostas das Unidades de Respostas Hidrológicas utilizado pelo modelo SWAT na simulação hidrossedimentológica.

\section{Conclusões}

As URHs com classes de solos NEOSSOLO LITÓLICO (RLve) e CAMBISSOLO HÁPLICO (CXve2) estão entre as que mais produzem sedimentos, evidenciando uma necessidade da prática de reflorestamento, pois ocorrem nas áreas de maior declividade da bacia do córrego Santa Maria.

Nas URHs sobre ARGISSOLOS VERMELHOAMARELOS (PVad) e os CAMBISSOLOS HÁPLICOS (CXve3 e CXve1), faz-se necessária a introdução de práticas de manejo e conservação do solo nas áreas de pastagem, visando minimizar a produção de sedimentos pela erosão hídrica e a ocorrência de feições erosivas.

O COE obtido após a calibração do modelo SWAT indica um ajuste ruim do modelo à bacia do córrego Santa Maria, o que, provavelmente, está associado aos dados utilizados para calibração e validação do modelo, indicando a necessidade de séries de dados mais robustas para modelagem hidrossedimentológica.

Apesar do baixo COE obtido neste trabalho, à modelagem da produção de sedimentos com o modelo SWAT mostra-se como uma importante ferramenta para o planejamento ambiental, subsidiando medidas efetivas por parte dos órgãos responsáveis. 


\section{Agradecimentos}

Ao projeto "Planejamento conservacionista das terras e modelagem preditiva de sistemas aquíferos do cristalino, para recarga hídrica em bacias hidrográficas de relevo acidentado", financiado pelo "Programa de Apoio ao Desenvolvimento de Tecnologias Agropecuárias para o Brasil (PRODETAB)", liderado pela Empresa Brasileira de Pesquisa Agropecuária (Embrapa) do Estado do Rio de Janeiro, que forneceu grande parte dos dados obtidos na bacia do córrego Santa Maria, que foram utilizados no presente estudo.

Ao Instituto Nacional de Meteorologia (Inmet - Brasília) pela concessão dos dados climáticos.

Ao Conselho Nacional de Ensino e Pesquisa (CNPq) pela concessão da bolsa de doutorado.

\section{Referências Bibliográficas}

AKSOY, H.; KAVVAS, M. L. A review of hillslope and watershed scale erosion and sediment transport models. Catena, v. 64, n. 2-3, p. 247-271, 2005.

ARAÚJO-NETO, M. D. Métodos de medição da água no solo: uma breve discussão. Geonômos, v. 2, n. 2, p. 51-61, 1993.

ARNOLD, J. G.; SRINIVASAN, R. A. Continuous catchmentscale erosion model. In: BOARDMAN, J.; FAVIS-MORTLOCK, D. (Ed.). Modelling soil erosion by water. Oxford. Publisher Sprringer, 1995. p. 413 - 428.

ARNOLD, J. G. et al. SWRRB: A basin scale simulation model for soil and water resources management. Texas: Press College Station. 1990. 255p.

BACCARO, C. A. D. Processos erosivos no domínio do Cerrado. In: GUERRA, A. J. T.; SILVA, A. S.; BOTELHO, R. G. M. Erosão e conservação dos solos. Rio de Janeiro: Editora Bertrand Brasil, 1999. p. 195- 228.

BEASLEY, C. B. et al. ANSWERS: a model for watershed planning. Trans. Am. Soc. Agric. Eng., v. 23, n. 8, p. 938-944, 1980.

BEVEN, K. J. Rainfall-runoff modelling. New York: John Willy \& Sons. 2001. 360 p.

BHERING, S. B. et al. Caracterização edafoambiental das microbacias de Cambiocó e Santa Maria no Município de São José de Ubá, região noroeste do Estado do Rio de Janeiro para fins de planejamento conservacionista. In: CONGRESSO BRASILEIRO DE CIÊNCIA DO SOLO (CBCS): solos, sustentabilidade e qualidade ambiental, 30.,2005, recife. Anais.... Recife: Embrapa Solos (UEP), 2005. 1 cd-Rom.

BOIX-FAYOS, C. et al. Measuring soil erosion by field plots: understanding the sources of variation. Earth-Science Reviews, n. 78, p. 267-285, 2006.

CARSON, M. A., KYRKBY, M. J. Hillslope form and process. London: Press Cambridge University, 1972. 475 p.
CERDAN, O. et al. Scale effect on runoff from experimental plots to catchments in agricultural areas in Normandy. Journal of Hydrology, M. 299, p. 4-14, 2004.

COELHO NETO, A. L. Hidrologia de encosta na interface com a geomorfologia. In: GUERRA, A. J. T.; CUNHA, S. B. (Ed.). Geomorfologia: uma atualização de bases e conceitos. Rio de Janeiro: Editora Edgard Blücher, 1995. p. 93-148.

COELHO NETO, A. L. Overlandflow production in a tropical rainforest catchment: the role of the liter cover. Catena, v. 14, n. 3, 1987. p. 213-231.

DANTAS, M. E. Geomorfologia do Estado do Rio de Janeiro: Texto explicativo do mapa geomorfológico do Estado do Rio de Janeiro na escala 1:50.000. CPRM/DRM. Rio de Janeiro: CPRM/DRM, 2000. 60 p.

DE PLOEY, J.; GABRIELS, D. Medición de la pérdida del suelo y estudios experimentales. In: KIRKBY, M. J.; MORGAN, R. P. C., Erosión de suelos. México. Editorial Limusa, 1980. p 89-139.

De VENTE, J. POESEN, J. Predicting soil erosion and sediment yield at the basin scale: scale issues and semi-quantitative models. Earth-Science Reviews, n. 71, p 95-125. 2005.

DI LUZIO, M. et al. ArcView interface for SWAT2000: user's guide. Texas: Blackland Research Center, 2002. 337p.

EVANS, R. Mechanics of water erosion and their spatial and temporal controls: an empirical viewpoint. In: KIRKBY, M. J.; MORGAN, R. P. C. (Ed.). Soil erosion. Chichester, Cambridge: John Wiley and Sons Ltda, 1980. p.109-129.

FIDALGO, E. C. C.; ABREU, M. B. Uso de imagens Áster para o mapeamento do uso e cobertura da terra na bacia hidrográfica do rio São Domingos, RJ. In: SIMPÓSIO BRASILEIRO DE SENSORIAMENTO REMOTO, 17., 2005, Goiânia. Anais..... Goiânia (GO), 2005. p 3747- 3753.

FOX, H.R. et al. Soil erosion and reservoir sedimentation in the High Atlas Mountains, southern Morocco. In: WALLING, D.E.; PROBST, J.L. (Ed.). Rabat symposium, Rabat: IAHS Publication, 1997. p. 233-240.

FLANAGAN, D.C.; GILLEY, J. E.; FRANTI, T. G. Water erosion prediction project (WEPP): development history, model capabilities and future enhancements. Transactions of the ASABE, v. 50, n. 5, p 1603-1612, 2007.

GONÇALVES, A. O. et al. Caracterização climática da bacia do rio São Domingos. Workshop de integração de informações obtidas no âmbito do projeto Prodetab Aquíferos: Embrapa solos. 2006, Rio de Janeiro. Anais.... Rio de Janeiro (RJ), 2006, $1 \mathrm{~cd}-$ Rom.

GUERRA, A. J. T. Processos erosivos nas encostas. In: GUERRA, A. J. T.; CUNHA, S. B. (Ed.). Geomorfologia: uma atualização de bases e conceitos. Rio de Janeiro: Editora Bertrand Brasil, 1995. p. 149-210. 
HADLEY, R. F. et al. Recent development in erosion and sediment yield studies. Paris: United Nations Educational Scientific and cultural Organization - Unesco, 1985. 127 p.

HEILBRON, M. D. B. P. et al. Geologia da bacia do rio São Domingos, São José de Ubá, Rio de Janeiro. Boletim de Resumos do $9^{\circ}$ Simpósio de Geologia do Sudeste (SBG), Niterói (RJ). 2005. 118 p.

HEWAWASAM, T. et al. Increase of human over natural erosion rates in tropical highlands constrained by cosmogenic nuclides. Geology, n. 31. p. 597-600, 2003.

KNISEL, W. G. CREAMS: a field scale model for chemicals, runoff and erosion from agricultural management systems. U.S. Department of Agriculture, Science and Education Administration, Conservation Research Report n 26. 1980. 643p.

LANE, L.J. et al. Processes controlling sediment yield from watersheds as function of spatial scale. Environmental Modelling and Software, v. 12, n. 4, p. 355-369, 1997.

MARCHIORO, E. Modelagem hidrossedimentológica na bacia do córrego Santa Maria: subsídios à aplicação de práticas de conservação de água e solo no noroeste fluminense. 2008, 196 f. Tese (Doutorado) - Programa de Pós-Graduação em Geografia/ Universidade Federal do Rio de Janeiro, Rio de Janeiro, 2008.

MARCHIORO, E.; AUGUSTIN, C. H. R. R. Dimensão de parcelas experimentais: influência nas medidas de escoamento superficial e erosão do solo em Gouveia/MG. Revista Geografia. Revista do Departamento de Geografia e do Programa de PósGraduação em Geografia (IGC/UFMG), Belo Horizonte, v. 3, n. 2, p. 7-16, Julho-Dezembro, 2007.

MERRITT, W. S.; LETCHER, R. A.; JAKEMAN, A. J. A review of erosion and sediment transport models. Environmental Modelling and Software, n. 18, p 761-799, 2003.

MINOTTI, R. T. Abordagens qualitativas e quantitativas de micro-bacias hidrográficas e áreas alagáveis do médio Mogisuperior - SP. 2006. 231 f. Tese (Doutorado) - Programa de Pós-Graduação em Ciências da Engenharia Ambiental. Escola de Engenharia de São Paulo - USP, São Carlos, 2006.

MORGAN, R. P. C. Topics in applied geography. New York: Publisher Longman, 1980. 113 p.

NEITSCH, S. L. et al. Soil and water assessment tool: theoretical documentation - version 2005. Texas: Blackland Research Center, 2005. 525 p.

REIS, A. P.; MANSUR, K. L. Sinopse geológica do Estado do Rio de Janeiro na escala 1:400.000. Departamento de Recursos Minerais (DRM). Secretaria do Estado de Meio Ambiente do Rio de Janeiro. Nitéroi/RJ. 1995. 60 p.

STROOSNIJDER, L. Measurement of erosion: Is it possible? Catena, v. 64, p 164-173, 2005.

VANACKER, V. et al. Spatial variation of suspended sediment concentrations in a tropical Andean river system: the Paute River, southern Ecuador. Geomorphology, v. 87, p. 53-67, 2007.

WILLIAMS, J. R. Chapter 25: The EPIC Model. In: SINGH, V. P. (Ed.). Computer models of watershed hydrology. Littleton: Water Resources Publications, 1995. p. 909-1000.

WILLIAMS, J. R.; JONES, C. A.; DYKE, P. T. A modeling approach to determining the relationship between erosion and soil productivity. Trans. ASAE, v. 27, n. 1, p. 129-144, 1984.

WILLIAMS, J. R.; NICKS, A. D.; ARNOLD, J. G. Simulator for waters resources in rural basins. Journal Hydraulic Eng., v. 111, p. 970-976, 1985.

ZARONI, M. J. Estimativa da produção de sedimentos em bacias hidrográficas por meio do modelo de erosão USLE e do índice de transferência de sedimentos - SDR. 2006, 143 f. Dissertação (Mestrado) - Programa de Pós-Graduação em Geografia (UFRJ), Rio de Janeiro (RJ), 2006. 